\title{
Adipose tissue remodeling and obesity
}

\author{
Kai Sun, ${ }^{1}$ Christine M. Kusminski, ${ }^{1}$ and Philipp E. Scherer ${ }^{1,2}$ \\ ${ }^{1}$ Touchstone Diabetes Center, Department of Internal Medicine, and 2Department of Cell Biology, \\ University of Texas Southwestern Medical Center, Dallas, Texas, USA.
}

\begin{abstract}
To fulfill its role as the major energy-storing tissue, adipose has several unique properties that cannot be seen in any other organ, including an almost unlimited capacity to expand in a non-transformed state. As such, the tissue requires potent mechanisms to remodel, acutely and chronically. Adipocytes can rapidly reach the diffusional limit of oxygen during growth; hypoxia is therefore an early determinant that limits healthy expansion. Proper expansion requires a highly coordinated response among many different cell types, including endothelial precursor cells, immune cells, and preadipocytes. There are therefore remarkable similarities between adipose expansion and growth of solid tumors, a phenomenon that presents both an opportunity and a challenge, since pharmacological interventions supporting healthy adipose tissue adaptation can also facilitate tumor growth.
\end{abstract}

\section{Introduction}

Adipose tissue (AT) can respond rapidly and dynamically to alterations in nutrient deprivation and excess through adipocyte hypertrophy and hyperplasia, thereby fulfilling its major role in wholebody energy homeostasis. AT remodeling is an ongoing process that is pathologically accelerated in the obese state, and thus, features such as reduced angiogenic remodeling, ECM overproduction, a heightened state of immune cell infiltration and subsequent proinflammatory responses prevail in many obese fat-pads (1). However, not all AT expansion is necessarily associated with pathological changes. The concept of the "metabolically healthy obese" state (2) suggests that some individuals can preserve systemic insulin sensitivity on the basis of "healthy" AT expansion, bypassing all of the aforementioned pathological consequences associated with obesity (3), thereby also avoiding the obesity-associated lipotoxic side effects. Many physiologically relevant processes important for human AT remodeling can be studied in rodent models, with the added advantage that processes related to AT expansion and reduction can occur at an extremely rapid rate. A 24-hour fast in a mouse is associated with a dramatic loss of AT mass and an acute remodeling process that involves rapid infiltration of macrophages; moreover, merely 24 to 48 hours of exposure to a high-fat diet (HFD) can cause a prompt increase in adipocyte size (4). AT is therefore an ideal model system to study rapid alterations in tissue expansion and reduction, as it adapts to a differential nutrient supply. Here, we will focus on key aspects of the intricate dynamics of AT remodeling and subsequent inflammatory consequences that arise from obesity.

\section{Macrophages, major constituents of AT and mediators of remodeling}

In 2003, two independent articles in the JCI highlighted the infiltration of macrophages into expanding AT as an important physiological phenomenon $(5,6)$. While not only opening up a newfound fundamental role for the macrophage in metabolism, these reports also fueled the publication of numerous additional papers, thus firmly establishing the phenomenon of a macrophage-orchestrated inflammatory response co-existing with obesity-induced insulin resistance.

Authorship note: Kai Sun and Christine M. Kusminski contributed equally to this work.

Conflict of interest: The authors have declared that no conflict of interest exists. Citation for this article: J Clin Invest. 2011;121(6):2094-2101. doi:10.1172/JCI45887.
Supporting this hypothesis, large clusters of macrophage-related inflammatory genes were identified as significantly altered in obese AT (5). Rodent studies revealed increased levels of macrophage markers present in AT of leptin-deficient, leptin receptor-deficient and diet-induced obese (DIO) mouse models (5). In parallel, obese subjects were found to harbor increased AT macrophage (ATM $\Phi)$ content, particularly in more insulin-resistant visceral depots (7). Conversely, weight reduction regimens resulted in a lowering of inflammatory markers, which suggested that this infiltration is reversible (7).

\section{The "initiation" of macrophage infiltration into adipose:} four different mechanisms

Adipocyte hyperplasia and hypertrophy can both contribute to AT expansion. This expansion can lead to a myriad of effects, including hypoxia, adipocyte cell death, enhanced chemokine secretion, and dysregulation in fatty acid fluxes (8). Such adipocyterelated consequences pose major logistical challenges and require macrophages to create a permissive environment for the remodeling process. Adipocytes are therefore at the very core of initiation of macrophage recruitment.

Adipocyte death. As "professional" phagocytes, macrophages are extremely proficient in the removal of numerous molecules, ranging from small lipids to colonies of pathogens to dead cells (9). Cinti and colleagues hypothesized that necrosis of adipocytes, driven by hypertrophy and accelerated by obesity, is a prominent phagocytic stimulus that regulates ATM $\Phi$ infiltration (10). Indeed, macrophages have been shown to aggregate, forming crown-like structures (CLSs) surrounding necrotic adipocytes in advanced obesity (10-13). In this state, they fuse to phagocytose the residual lipid droplet, forming large lipid-laden multinucleated syncytia in the process, a commonly accepted hallmark of chronic inflammation $(10,14)$. Using a transgenic model of inducible lipoatrophy, we have previously demonstrated that massive adipocyte death can indeed drive rapid accumulation of ATMФs as an integral element in the remodeling of fat pads (15). While we used a model of inducible apoptosis in this particular case, the apoptosis in our model recapitulates the necrosis observed by Cinti and colleagues (10) in many aspects. It is almost impossible to determine whether adipocytes in vivo preferentially undergo a simple necrotic death or apoptosis, due to the technical difficulties of reproducibly demonstrating apoptosis in adipocytes. However, the death of an adipocyte, necrotic or apoptotic, leaves an inert 
lipid droplet behind that is devoid of the protein coat usually associated with an intracellular lipid droplet, as well as devoid of any lipases that could mediate hydrolysis of triglycerides. The removal of these lipid droplets is an integral aspect of AT remodeling. If the situation in the inducible lipodystrophy model in the mouse is an indication of the rate of lipid droplet removal under normal physiological conditions, then this is likely a relatively slow process, occurring over a period of days to weeks (15).

Chemotactic regulation. Chemokines are small proinflammatory molecules that promote macrophage mobilization from bone marrow into tissues. There is considerable evidence for the pathophysiological role of macrophage- and/or hypertrophic adipocytederived chemotactic MCP-1/CCR2 pathways in the regulation of monocyte accumulation in obese $\operatorname{AT}(16,17)$. In particular, increased expression levels of MCP-1, CXCL14, MIP-1 $\alpha$, MCP-2, MCP-3, and RANTES can be observed in AT of mice with genetic or $\operatorname{DIO}(5,16,18)$.

Hypoxia. Adipocyte hypertrophy creates areas of local AT microhypoxia at the earliest stages of expansion (19). This has been verified by recent clinical observations in humans, which suggest that AT is poorly oxygenated in the obese state $(20,21)$. The effects of the local AT hypoxia have been investigated both in isolated murine adipocytes and in animal models (4, 22-25). These papers suggest that many adipokines that are related to inflammation, such as macrophage migration inhibitory factor (MIF), the matrix metalloproteinases MMP2 and MMP9, IL-6, Angplt4, PAI-1, VEGF, and leptin are all upregulated by hypoxia (22-25).

A master regulator of hypoxia and oxygen homeostasis is HIF-1 $(26,27)$. Several important hypoxia-associated genes, such as leptin and VEGF, are directly regulated by HIF-1 (25). As a transcription factor, HIF-1 functions as a heterodimer, consisting of an oxygensensitive HIF- $1 \alpha$ subunit and a constitutively expressed HIF-1 $\beta$ subunit. While HIF- $1 \alpha$ synthesis is $\mathrm{O}_{2}$ independent, its degradation is enhanced under normoxic conditions (28). As such, $\mathrm{O}_{2}$ can mediate the prolyl hydroxylation of HIF-1 $\alpha$, which subsequently facilitates ubiquitination by E3 ligase. This ligase contains a von Hippel-Lindau (VHL) tumor-suppressor protein, which specifically recognizes the hydroxylated form of HIF- $1 \alpha$ and targets it for ubiquitination and degradation.

To investigate the effects of HIF- $1 \alpha$ in white AT, we analyzed a transgenic mouse model in which we overexpressed a dominant active (degradation-resistant) deletion mutant of HIF-1 $\alpha$ (HIF- $1 \alpha-$ $\Delta$ ODD) specifically in adipocytes (4). Unexpectedly, our model failed to induce any classical HIF-1 $\alpha$ targets, such as VEGF-A. Moreover, we did not detect any increases in key components of the angiogenic or anaerobic glycolytic programs (4). The most striking alteration we observed in this model was an enhanced fibrotic response, which included an upregulation lysyl oxidase (LOX), elastin, collagens I and III, tissue inhibitor of metalloproteinase I, and connective tissue growth factor. As the result of this aberrant HIF-mediated transcriptional program, we proposed that the ECM in white AT accumulates fibrillar collagens; the upregulation of ECM thus causes local fibrosis. Collectively, our results suggest that hypoxia-induced fibrosis in AT may be a key factor that ultimately stimulates the local inflammatory responses.

Fatty acid flux. FFAs, stored in the form of triglycerides in AT, are released from hypertrophic adipocytes through lipolysis during fasting. Some of these FFAs are shunted to the liver and stored in lipid droplets, while some of them are oxidized in other organs. However, many of them are locally re-esterified in adipocytes
(29-31). Those FFAs that escape reesterification play a critical role in several organs as a primary energy source during prolonged fasting (32). However, FFAs can also serve as ligands for the TLR4 complex (33), thereby activating the classical inflammatory response in the context of increased local extracellular lipid concentrations, which ultimately drives ATM $\Phi$ accumulation (34, 35 ). Even though we do not consider AT as a conventional tissue suffering from lipotoxic side effects, either high rates of lipolysis or an influx of saturated FFAs into adipocytes do cause temporary inflammation within the tissue (33).

While these four mechanisms of macrophage recruitment and infiltration into AT may act independently, the metabolic and inflammatory pathways are tightly interconnected. Future studies should reveal the precise temporal steps required for macrophage recruitment to AT in expanding fat pads.

\section{AT remodeling: the crosstalk between adipocytes and macrophages}

Although the adipocyte is the key player orchestrating local changes in the microenvironment, much evidence also points toward pivotal role for macrophages in such remodeling events. Resident ATM $\Phi$ s display remarkable heterogeneity in their activities and functions (36), largely reflecting the complex events occurring in AT during metabolic and immune perturbations.

The obesity-driven phenotypic switch in ATMФs. ATMФs can be characterized in two broad classes based on the expression of particular antigens $(37,38)$. In 2007, Saltiel and colleagues proposed a model of "phenotypic switching" that captured the very essence by which enhanced ATM $\Phi$ infiltration exacerbates the milieu of obesity-related inflammation (38). Their model emphasized that obesity is accompanied by a transformation in the polarized states of macrophages, from an antiinflammatory "alternatively activated" M2 form that primarily accumulates during negative energy balance (39), to a more proinflammatory "classically activated” M1 form (38). The M1 population demonstrates a positive correlation with insulin resistance and dominates in states of overnutrition by targeting FFA-mediated increases in proinflammatory responses $(14,40)$.

In the lean state, resident macrophages are polarized toward an M2 status. A hallmark feature of the M2 population is that they express F4/80, CD301, IL-10, and arginase $1(37,38)$, the latter of which has been shown to inhibit NOS (iNOS) activity (41). Such a combination of factors may help to preserve normal adipocyte function by promoting tissue repair and angiogenesis in the increasing AT mass (42). Conversely, M1 macrophages, induced by LPS and the Th1 cytokine IFN- $\gamma$, express a repertoire of proinflammatory factors, which include F4/80, CD11c, TNF- $\alpha$, IL-6, iNOS, and CCR2 $(14,38)$.

In light of this, Patsouris and colleagues utilized a conditional cell-ablation system based on the transgenic expression of the diphtheria toxin receptor, under the control of the CD11c promoter, to specifically ablate $\mathrm{CD} 11 \mathrm{c}^{+}$macrophages in the AT of HFD-fed obese mice (43). Such conditional ablation resulted in a profound reduction of proinflammatory ATM accumulation, accompanied by rapid whole-body normalization of insulin sensitivity (43). This further highlights the direct contribution of these cells toward local and systemic insulin sensitivity.

Taken together, these studies suggest that a delicate balance of polarized populations of macrophages is necessary to maintain adequate adipocyte function. Identifying factors that quench 


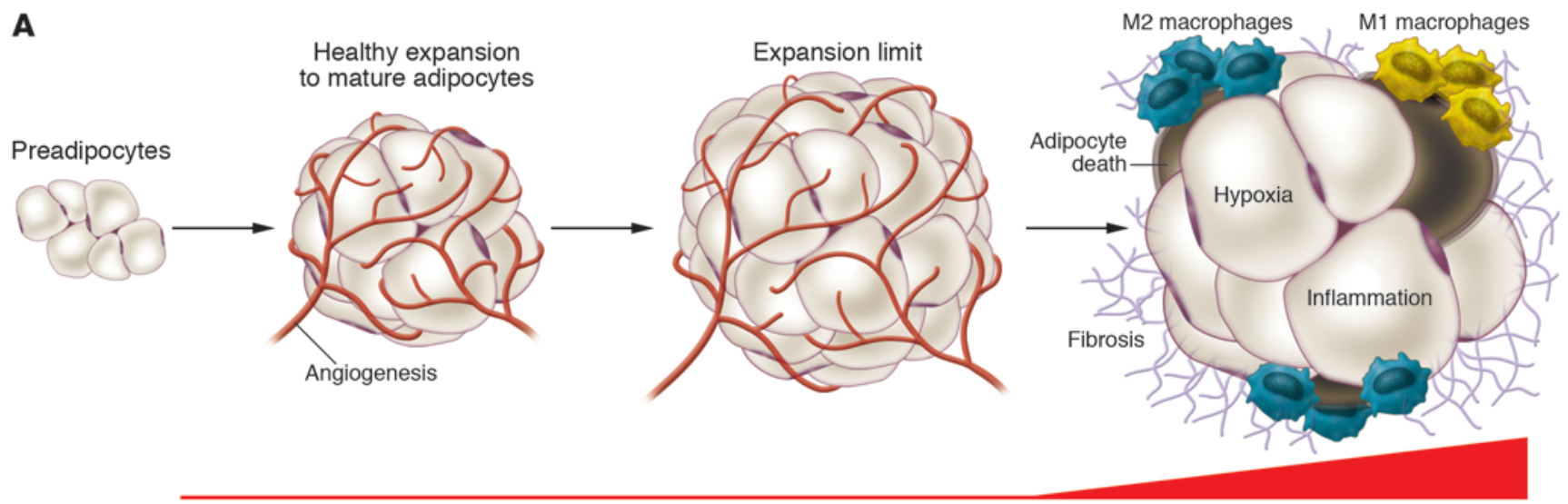

Insulin resistance

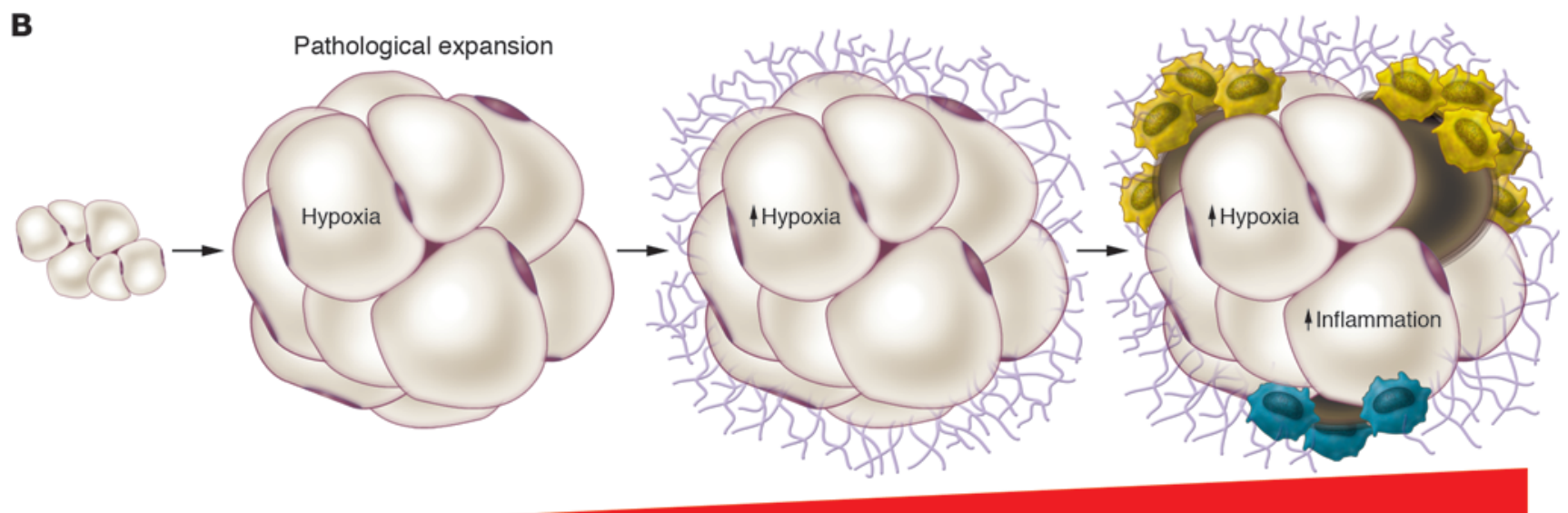

Insulin resistance

Figure 1

Healthy and unhealthy AT expansion. (A) Healthy AT expansion consists of an enlargement of AT through effective recruitment of adipogenic precursor cells to the adipogenic program, along with an adequate angiogenic response and appropriate remodeling of the ECM. There are strong individual differences with respect to the potential for AT expansion. (B) In contrast, pathological AT expansion consists of massive enlargement of existing adipocytes, limited angiogenesis, and ensuing hypoxia. As a result, HIF-1 $\alpha$ is induced, which in turn can cause the induction of a fibrotic program. Ultimately, M1-stage macrophages prevail, leading to an inflammatory phenotype that is strongly associated with systemic insulin resistance.

inflammatory signals in AT through modification of ATMs to retain an $\mathrm{M} 2$ polarization, or by triggering the phenotypic switch from M1 to M2, may be beneficial to preserve adequate adipocyte function and insulin action in an obese setting (Figure 1).

An important distinction needs to be established between healthy fat pad expansion and pathological fat pad expansion. We define healthy expansion as an enlargement of the fat pad mass through enhanced recruitment of adipocyte precursor cells that are differentiated into small adipocytes, along with the recruitment of other stromal cell types with appropriate ratios, and subsequent vascularization, minimal induction of ECM and minimal inflammation. In contrast, pathological expansion of AT can be described by rapid growth of the fat pad through enlargement of existing fat cells, a high degree of macrophage infiltration, limited vessel development, and massive fibrosis (ref. 8 and Figure 2). Such pathological expansion is associated with chronic inflammation, which ultimately results in the development of systemic insulin resistance.

\section{The intricate dynamics of AT remodeling}

Acute remodeling in context of weight loss. Recently, Kosteli and colleagues demonstrated that acute weight loss is unexpectedly associated with a rapid - albeit transient - lipolytic-driven recruitment of macrophages into AT (39), thereby confirming similar reports by Granneman and colleagues (44).

In contrast to the obese state, in which the occurrence of increased fat mass, adipocyte hypertrophy, and elevated basal lipolytic rates exacerbate the invasion of classically activated CD11 $\mathrm{c}^{+}$macrophage populations that permanently reside in the inflamed AT, we anticipate that remodeling due to weight reduction would lower ATM $\Phi$ content and subsequent inflammation. This was indeed observed by Kosteli et al. during chronic weight loss, during which they report that a progressive decrease in lipolysis and ATM $\Phi$ content followed 21 days of caloric restriction (39); $\mathrm{F} 4 / 80^{+}$and $\mathrm{CD}_{11} \mathrm{~b}^{+}$macrophages were further shown to be prominent during times of negative energy balance (39). Ironically, acute weight loss (days 0-7), a 24-hour fast, or pharmacological induction of 
A

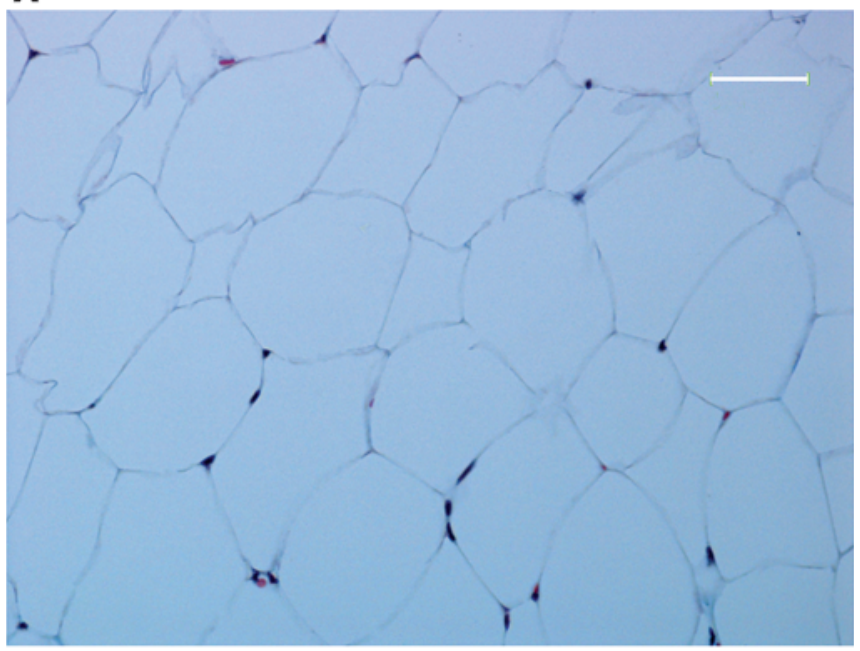

B

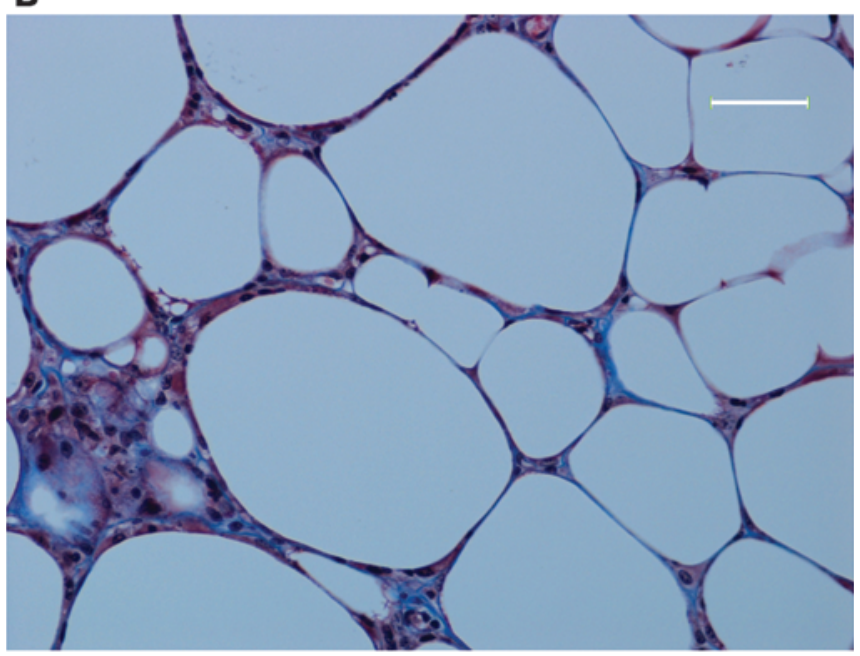

Figure 2

Healthy and unhealthy AT. (A) Trichrome stain of a healthy murine epididymal fat pad with densely packed, hexagonal adipocytes and limited immune cell infiltration. (B) Trichrome stain of an unhealthy ob/ob epididymal fat pad, containing a high level of immune cell infiltration and enhanced ECM (blue areas). Scale bars: $50 \mu \mathrm{m}$.

adipocyte lipolysis using a $\beta 3$-adrenergic agonist all resulted in a transient accumulation of lipid-laden ATMФs (an increase of up to $65 \%$ of macrophage infiltration was observed). This was reflected by an increase in the levels of macrophage-specific genes, F4/80, CD38 and CSF-R1 (39). Collectively, these studies suggest that a transient influx of ATMФs is a physiologically healthy response and further implicates a maladaptive inflammatory reaction in the chronic state. In addition, a chronic negative energy balance, which results in the mobilization of triglyceride stores through enhanced lipolytic rates, acts as a signal to circulating monocytes, providing an excellent paradigm for the adaptations and the beneficial interplay between adipocytes and macrophages that arise as a consequence of a reduction in AT mass. While we appreciate that during this process the egression of ATM $\Phi$ s from the adipose back into circulation following a period of fasting is fully effective, it is not known how we can prevent the lipid-laden proinflammatory $\mathrm{ATM} \Phi$ s from permanently residing in $\mathrm{AT}$ in the obese state. When and how do we transition from a physiologically healthy adaptive immune response to a pathological maladaptive state?

Understanding how AT remodels and acclimatizes to an acutely altered nutrient environment may provide key mechanistic insights into solving the puzzle of chronic ATM $\Phi$ recruitment into AT.

Chronic remodeling of AT: the adipocyte in the spotlight. With an exceptionally high level of adipokine production, it is legitimate to view the adipocyte as a "professional" secretory cell. In times of sustained excess of caloric intake, adipocytes undergo hypertrophy and hyperplasia, thus setting into motion the dynamic events of AT expansion. Hypertrophy results in an altered complement of secretory products, which over time adversely affects the remodeling of AT (45). In parallel, the hypertrophy provides valuable protection against the detrimental systemic lipotoxic effects of excess lipid exposure. These events entail mechanical stress through increased ECM overproduction, limited angiogenesis, and the induction of local inflammation, all of which continuously compromise the functional integrity of AT $(46,47)$.

Hypertrophy versus hyperplasia? Two distinct mechanisms can lead to increased AT size: hypertrophy (an increase in adipocyte volume) or hyperplasia (an increase in adipocyte cell number). Although we recognize that adipocyte hypertrophy prevails in obesity, there remains some debate as to whether the adipocyte number remains constant in an adult individual, or whether the ability to undergo hyperplasia is age dependent. Key questions to take into consideration are: what is the actual half-life of an adipocyte? Can certain processes alter the rate of adipocyte turnover? And if so, is the adipocyte indeed programmed to survive for a certain period of time, or can its fate be derailed under extreme metabolic oscillations?

Using incorporation of environmental ${ }^{14} \mathrm{C}$ as a tracer, Spalding and colleagues documented that "new adipocytes form constantly to replace lost adipocytes" and estimated the half-life of the average adipocyte to be in the order of 8.3 years (48). This group postulated that adipocyte cell number is relatively fixed by early adulthood, and that any alterations in fat mass during adulthood are merely credited to alterations in adipocyte hypertrophy (48). The rate of appearance of newly emerging adipocytes is balanced by adipocyte death, with the total number of adipocytes being tightly controlled, while the whole system is in a state of constant flux. This hypothesis suggests that adipocyte progenitor cells are either recruited into the stromal vascular fraction of adult AT (49) or propagated as precursor cells into mature AT, thus allowing them to differentiate into lipid-laden mature adipocytes at the same rate that existing adipocytes undergo cell death.

These studies, however, raise further questions: given that obesity is associated with a higher adipocyte turnover due to an elevated rate of cell death (10), are there conditions in which a higher rate of preadipocyte recruitment, rather than loss of mature adipocytes through cell death, can occur? Can we ever tip the balance to have an uneven "apoptotic-to-adipogenic ratio" (48), and what would be the metabolic consequences? Moreover, what are the signals that trigger an influx of preadipocytes and enhanced recruitment of preadipocytes towards the adipogenic axis? One plausible mechanism may be that once adipocytes reach a critical volume, they secrete factors that recruit new adipocytes (50); however, the details of these processes are far from understood.

The effect of age on adipocytes and AT remodeling has also recently been the focus of several studies. Adipogenesis and the 
intrinsic functional dynamics of preadipocytes en route to develop into fully functional mature adipocytes have been documented to decline markedly with age $(51,52)$. In particular, the capacity of preadipocytes to proliferate, differentiate, and confer resistance to cell death is severely blunted with age $(51,53)$. As such, the inherent characteristics of these cells are shown to contribute to age- and depot-dependent variations in AT function, collectively resulting in metabolically unfavorable ectopic fat accumulation $(51,54,55)$.

Is the macrophage involved in adipocyte turnover? Strissel and colleagues provided key answers to this question by assessing the progression of adipocytes and macrophages in AT of HFD-fed mice. As expected from previous studies, the group initially observed that following 16 weeks of HFD feeding, a high degree (approximately $80 \%$ ) of adipocyte cell death was evident in epididymal AT (13). However, a fascinating twist to these findings was that at the 20-week stage, the percentage of necrotic cells was reduced to approximately $16 \%$; this was accompanied by an increased emergence of smaller adipocytes $\left(<5,000 \mu \mathrm{m}^{2}\right)$, thus restoring the adipocyte number (13). These key findings lend support to a model in which the progression of obesity is associated with AT remodeling, expanding largely through hypertrophy, subsequently requiring adipocytes to undergo an initial "necrotic wave," with later stages relying on hyperplastic expansion.

So, where do ATMФs fit into this equation of adipocyte turnover and AT expansion? And does a macrophage polarization shift occur in response to fluctuations in adipocyte turnover? The initial surge of cell death was shown to be associated with the presence of M1-like ATM $\Phi$ s. In contrast, progressing on to the hyperplastic stage of AT transition, a reduction in M1-type markers was evident with a concomitant appearance of M2 macrophages (13).

Other immune cells: the emerging physiological role of T cells in AT physiology. In addition to macrophages, recent studies have revealed a growing list of other immune cells that may orchestrate AT remodeling. These include subsets of the $\mathrm{T}$ lymphocyte lineage, including $\mathrm{CD}^{+}$and $\mathrm{CD}^{+} \mathrm{T}$ cells, Tregs, and mast cells $(56,57)$. In particular, levels of a specific subpopulation of $\mathrm{CD}^{+} \mathrm{T}$ cells are enriched in the early stages of obesity, in fact preceding the accumulation of ATMФs (56). This certainly implicates a potential role for $\mathrm{CD}^{+}$ $\mathrm{T}$ cells in the initiation of the subsequent inflammatory cascade. Mast cells have recently been highlighted as another AT-associated cell type; abundant in obese AT, these inflammatory cells may elicit a pathogenic role in AT functionality (58). As a whole, these studies underscore that, in order to design therapeutic strategies, it is important to understand the imbalance of Tregs in AT during the progression of inflammation in expanding AT.

\section{Angiogenesis, a rate-limiting step for AT expansion and remodeling}

Angiogenesis is the process of formation of new blood vessels. Tumor expansion critically depends on an appropriate blood supply, which can be provided by new blood vessels from surrounding tissues. Angiogenesis plays a critical role in tumor growth and metastasis formation, and blocking angiogenesis prevents tumor growth and forces regression of existing lesions (59-61). However, in healthy adults, the majority of tissues do not expand in size, and the embedded blood vessels are thus quiescent (62). One notable exception is AT, which displays by nature a higher level of plasticity and retains the potential to grow throughout the entire lifetime. AT can rapidly expand or regress under different nutritional conditions; therefore newly emerging adipocytes are highly angiogenic. The angiogenic activity of white AT has been demonstrated in a number of in vitro systems, and has been further utilized clinically to promote wound healing (63). The newly formed vascular network is crucial for adipogenesis and AT expansion $(64,65)$. These vessels provide $\mathrm{O}_{2}$, nutritional components, growth factors, hormones, inflammatory cells, and bone marrow-derived stem cells to maintain adequate homeostasis of AT, all of which are crucial for further expansion (66). Effective development of the vascular supply through angiogenesis is therefore a rate-limiting step in AT expansion (67).

Different fat pads vary with respect to their degree of angiogenic potential. The vascular density and abundance of endothelial cells is higher in visceral AT in comparison to subcutaneous fat pads (68). Moreover, endothelial cells in visceral AT exhibit more potent angiogenic and inflammatory properties (68).

During AT remodeling in metabolically challenging conditions, adipokines are subjected to differential regulation, thereby orchestrating the growth and expansion of various fat pads. Leptin can stimulate the critical steps required to evoke an angiogenic program (69). In addition, bFGF can promote vascular endothelial cell growth (70) and hence the process of angiogenesis. Of note, bFGF is secreted by preadipocytes, and its levels are increased during caloric excess. Moreover, upon proper nutritional stimulation, AT also synthesizes HGF and VEGF (71), both of which play key pro-angiogenic roles (71). The situation seems more complex for adiponectin, however. Some studies have suggested anti-angiogenic properties for adiponectin (72), but these studies rely on recombinant preparations of the protein, and the quality of these preparations varies widely. Other in vivo studies demonstrating adiponectin-mediated inhibition of angiogenesis in mouse cornea assays suffer from the same shortcomings (73). In contrast, data from several other more reliable in vivo studies suggest potent proangiogenic effects (74). Using a genetic approach, our laboratory identified that adiponectin has potent angiomimetic properties in tumor vascularization (75).

VEGF-A is the only bona fide endothelial cell growth factor; moreover, its presence is essential for initiation of the angiogenic program $(76,77)$. VEGF-A exhibits predominantly pro-angiogenic activity in AT (78). Furthermore, VEGF levels are known to be regulated by hypoxia, insulin stimulation, certain growth factors, and specific cytokines (79) and vary during adipogenesis (80). In brief, VEGF functions by binding two tyrosine kinase receptors, VEGFR1 and VEGF-R2; the latter is expressed in vascular endothelial cells, and signaling through this receptor is critical for both physiological and pathological blood vessel formation. Blockade of VEGF-R2, but not VEGF-R1, restricts AT expansion and thus prevents the progression of DIO (81). Remarkably, blockade through use of a VEGF-R2 antibody can further inhibit preadipocyte differentiation (82). Due to their dramatic effects in angiogenesis and hence AT remodeling, VEGF and its receptors should be considered as key targets worth further exploration in the context of development of pharmacological anti-obesity approaches.

The angiopoietin proteins are the most important functional partners of VEGF. Both Ang-1 and Ang- 2 can bind to the common endothelial cell tyrosine receptor, Tie- $2(83,84)$. More specifically, Ang- 1 is an agonist of the Tie- 2 receptor, which is constitutively expressed in several tissues, including AT (83). The activity of Ang-1 can enhance VEGF receptor function for vascular development, remodeling, stabilization, and final maturation (85-87). Ang-2, on the other hand, is an antagonist for the Tie-2 receptor and is exclu- 
sively expressed in sites of vascular remodeling $(83,84,88)$. In these regions, Ang- 2 can block the action of Ang-1 through competitive inhibition, thereby destabilizing the vessel structure (84). However, delineating an unequivocal definition of the precise function of Ang- 2 is proving somewhat challenging, since it very much depends on the context; for instance, in the presence of VEGF, Ang-2 can prime vessels to mount a robust pro-angiogenic response (89).

Several reports suggest a role of angiopoietins and Tie- 2 in AT, but to date, the functions of this family of proteins have not been directly examined. Xue and colleagues recently reported that AT FOXC2 can mediate certain aspects of angiogenesis and vascular patterning (90). Given that Ang-2 is an established transcriptional target of FOXC2 (90), it is likely that Ang-2 plays an important role in this pathway. When inhibiting the function of Ang-2 with a neutralizing antibody, the effects caused by FOXC2 are equally blocked (90). Further studies are therefore required to better define the precise function of angiopoietins in AT remodeling.

Promoting an angiogenic cascade in healthy fat pads can effectively counteract hypoxic conditions and could thus be associated with metabolically beneficial effects. Adiponectin, for example, has been shown to induce angiogenesis in hypoxic fat pads and tumors $(75,91)$. Moreover, we have previously demonstrated that overexpression of adiponectin in an $o b / o b$ background improves vascularization and expansion of the subcutaneous fat pad in particular (91).

$\mathrm{O}_{2}$ tension in the center of solid tumors may be so low that cells essentially become anoxic; this is recognized to have profound effects on the tumor metabolism (92-94). We have discussed hypoxia as a key player in expanding AT that serves as a driving force for macrophage infiltration. Compared to brown AT, white AT is not particularly well vascularized $(4,20)$. The $\mathrm{O}_{2}$ tension in obese white AT can reach levels as low as $15 \mathrm{mmHg}$, much lower than that in normal lean AT, in which values would typically reach $45-50 \mathrm{mmHg}$ (23). Moreover, the postprandial increase in blood flow that is so frequently observed in lean individuals is dramatically reduced in obese individuals $(95,96)$. Here we should take into consideration the large diameter that an individual adipocyte can acquire (150-200 $\mu \mathrm{m})$; this is markedly larger than the average diffusion distance of $\mathrm{O}_{2}$ in tissues (97). Several mouse models of hypoxia have been described in the literature $(4,22,23,98,99)$. We have also shown that exposure to a HFD for merely a few days results in a significant increase in adipocyte cell size; this consequently leads to an acute hypoxic response within the adipocytes themselves (4).

\section{The role of collagens and matrix metalloproteases in the remodeling process}

Adipocytes are enmeshed in a dense network of $\operatorname{ECM}(100,101)$. The ECM not only functions to provide mechanical support for a fat pad, but also regulates the physiological and pathological events of AT remodeling through a variety of signaling pathways (102). During AT expansion, the ECM actively remodels to accommodate the growth. We and others have demonstrated that several ECM components are upregulated during fat mass expansion in states of obesity (102, 103). AT fibrosis, with its associated reduced plasticity, is therefore a key hallmark of the metabolically dysfunctional AT. Collagen VI, for example, is a collagen complex that is highly enriched in the ECM of AT (102). We have demonstrated that the weakening of the ECM that surrounds AT by elimination of collagen VI leads to improved survival rates of adipocytes and improvements in metabolism (102). Others have further reported correlations between elevated collagen VI levels, hyperglycemia, and insulin resistance $(104,105)$.

The ECM components in AT are known to be regulated by matrix metalloproteinases, a family of neutral endopeptidases that cleave ECM components, thereby enabling remodeling of ECM (106). Chun and colleagues highlighted the essential role for one of these proteases by demonstrating that MT1-MMP (MMP14) has a direct impact on adipose differentiation in vitro and in vivo (101). The absence of MT1-MMP impairs white AT development and hence causes lipodystrophy (101). The function of several other MMPs highly expressed in AT remains to be further identified. Maquoi and colleagues further demonstrated that in DIO mice, expression levels of MMP3, $-11,-12,-13$, and -14 are all upregulated, whereas levels of MMP7, $-9,-16$, and -24 are downregulated (106). In parallel, specific tissue inhibitors of MMPs (TIMPs) can also be dysregulated. More specifically, TIMP1 is upregulated with obesity, and conversely, TIMP4 is downregulated in states of obesity (106). Such striking regulatory patterns of MMPs and their corresponding inhibitors suggest important roles of these factors in the pathophysiology of AT.

\section{Concluding remarks}

We underestimate the complex events that must occur in order for an organ such as AT to rapidly remodel and either release or accept a large number of calorically dense lipids that have the potential to be potently cytotoxic. A well-orchestrated set of interactions between a number of critical cell types has to take place in a defined chronological order, with many pathological changes that can occur during that process. While research in the past decade has focused on the macrophage as an important player in AT remodeling, future efforts will unquestionably address the unique properties of other cells, including AT-associated endothelial cells as key players for a healthy adipocyte microenvironment.

\section{Acknowledgments}

We would like to thank our colleagues in the Touchstone Diabetes Center for critical comments on the manuscript. Our work is supported by NIH grants R01-DK55758, R01-CA112023, RC1DK086629, and P01-DK088761 (to P.E. Scherer). C.M. Kusminski is supported by a fellowship from the Juvenile Diabetes Foundation (JDRF 3-2008-130). We would like to thank Nils Halberg for help with Figure 2. The Obesity Review Series is supported in part by unrestricted educational grants from Merck \& Co. and the Life Sciences Institute of the University of Michigan.

Address correspondence to: Philipp E. Scherer, Touchstone Diabetes Center, Department of Internal Medicine, University of Texas Southwestern Medical Center, 5323 Harry Hines Blvd., Dallas, Texas 75390-8549, USA. Phone: 214.648.8715; Fax: 214.648.8720; E-mail: Philipp.Scherer@utsouthwestern.edu.
1. Hotamisligil GS, Shargill NS, Spiegelman BM. Adipose expression of tumor necrosis factor-alpha: direct role in obesity-linked insulin resistance. Science. 1993;259(5091):87-91.

2. Ruderman NB, Schneider SH, Berchtold P. The "metabolically-obese," normal-weight individual.
Am J Clin Nutr. 1981;34(8):1617-1621.

3. Unger RH, Scherer PE. Gluttony, sloth and the metabolic syndrome: a roadmap to lipotoxicity. Trends Endocrinol Metab. 2010;21(6):345-352.

4. Halberg $\mathrm{N}$, et al. Hypoxia-inducible factor 1 alpha induces fibrosis and insulin resistance in white adi- pose tissue. Mol Cell Biol. 2009;29(16):4467-4483.

5. Xu H, et al. Chronic inflammation in fat plays a crucial role in the development of obesity-related insulin resistance. J Clin Invest. 2003;112(12):1821-1830.

6. Weisberg SP, McCann D, Desai M, Rosenbaum M, Leibel RL, Ferrante AW Jr. Obesity is associated 
with macrophage accumulation in adipose tissue. J Clin Invest. 2003;112(12):1796-1808.

7. Cancello R, et al. Increased infiltration of macrophages in omental adipose tissue is associated with marked hepatic lesions in morbid human obesity. Diabetes. 2006;55(6):1554-1561.

8. Sun K, Scherer PE. Adipose Tissue Dysfunction: A Multistep Process. Berlin, Germany: Springer-Verlag Berlin Heidelberg; 2010:67-75.

9. Gordon S. The macrophage. Bioessays. 1995 17(11):977-986.

10. Cinti S, et al. Adipocyte death defines macrophage localization and function in adipose tissue of obese mice and humans. J Lipid Res. 2005; 46(11):2347-2355.

11. Nishimura $\mathrm{S}$, et al. Adipogenesis in obesity requires close interplay between differentiating adipocytes, stromal cells, and blood vessels. Diabetes. 2007; 56(6):1517-1526

12. Nishimura $S$, et al. In vivo imaging in mice reveals local cell dynamics and inflammation in obese adipose tissue. J Clin Invest. 2008;118(2):710-721.

13. Strissel KJ, et al. Adipocyte death, adipose tissue remodeling, and obesity complications. Diabetes. 2007;56(12):2910-2918.

14. Lumeng CN, Deyoung SM, Bodzin JL, Saltiel AR. Increased inflammatory properties of adipose tissue macrophages recruited during diet-induced obesity. Diabetes. 2007;56(1):16-23.

15. Pajvani UB, et al. Fat apoptosis through targeted activation of caspase 8: a new mouse model of inducible and reversible lipoatrophy. Nat Med. 2005; 11(7):797-803

16. Kanda H, et al. MCP-1 contributes to macrophage infiltration into adipose tissue, insulin resistance, and hepatic steatosis in obesity. J Clin Invest. 2006; 116(6):1494-1505

17. Weisberg SP, et al. CCR2 modulates inflammatory and metabolic effects of high-fat feeding. $J$ Clin Invest. 2006;116(1):115-124.

18. Nara N, et al. Disruption of CXC motif chemokine ligand-14 in mice ameliorates obesity-induced insulin resistance. J Biol Chem. 2007;282(42):30794-30803.

19. Trayhurn P, Wood IS. Adipokines: inflammation and the pleiotropic role of white adipose tissue. $\mathrm{Br}$ J Nutr. 2004;92(3):347-355.

20. Kabon B, et al. Obesity decreases perioperative tissue oxygenation. Anesthesiology. 2004;100(2):274-280.

21. Virtanen KA, et al. Glucose uptake and perfusion in subcutaneous and visceral adipose tissue during insulin stimulation in nonobese and obese humans. J Clin Endocrinol Metab. 2002;87(8):3902-3910.

22. Hosogai N, et al. Adipose tissue hypoxia in obesity and its impact on adipocytokine dysregulation. Diabetes. 2007:56(4):901-911.

23. Ye J, Gao Z, Yin J, He Q. Hypoxia is a potential risk factor for chronic inflammation and adiponectin reduction in adipose tissue of ob/ob and dietary obese mice. Am J Physiol Endocrinol Metab. 2007; 293(4):E1118-E1128

24. Chen B, et al. Hypoxia dysregulates the production of adiponectin and plasminogen activator inhibitor-1 independent of reactive oxygen species in adipocytes. Biochem Biophys Res Commun. 2006;341(2):549-556.

25. Lolmede K, Durand de Saint Front V, Galitzky J, Lafontan M, Bouloumie A. Effects of hypoxia on the expression of proangiogenic factors in differentiated 3T3-F442A adipocytes. Int J Obes Relat Metab Disord. 2003·27(10):1187-1195.

26. Brahimi-Horn MC, Chiche J, Pouyssegur J. Hypoxia and cancer. J Mol Med. 2007;85(12):1301-1307.

27. Brahimi-Horn MC, Pouyssegur J. Oxygen, a source of life and stress. FEBS Lett. 2007;581(19):3582-3591.

28. Kaelin WG Jr, Ratcliffe PJ. Oxygen sensing by metazoans: the central role of the HIF hydroxylase pathway. Mol Cell. 2008;30(4):393-402.

29. Thompson BR, Lobo S, Bernlohr DA. Fatty acid flux in adipocytes: the in's and out's of fat cell lipid traf- ficking. Mol Cell Endocrinol. 2010;318(1-2):24-33.

30. Ahmadian M, Wang Y, Sul HS. Lipolysis in adipocytes. Int J Biochem Cell Biol. 2010;42(5):555-559.

31. Unger RH, Clark GO, Scherer PE, Orci L. Lipid homeostasis, lipotoxicity and the metabolic syndrome. Biochim Biophys Acta. 2010;1801(3):209-214.

32. Carmen GY, Victor SM. Signalling mechanisms regulating lipolysis. Cell Signal. 2006;18(4):401-408.

33. Shi $\mathrm{H}$, Kokoeva MV, Inouye $\mathrm{K}$, Tzameli I, Yin $\mathrm{H}$, Flier JS. TLR4 links innate immunity and fatty acid-induced insulin resistance. J Clin Invest. 2006; 116(11):3015-3025.

34. Suganami T, Nishida J, Ogawa Y. A paracrine loop between adipocytes and macrophages aggravates inflammatory changes: role of free fatty acids and tumor necrosis factor alpha. Arterioscler Thromb Vasc Biol. 2005;25(10):2062-2068.

35. Nguyen MT, et al. JNK and tumor necrosis factor-alpha mediate free fatty acid-induced insulin resistance in 3T3-L1 adipocytes. J Biol Chem. 2005; 280(42):35361-35371.

36. Gordon S, Taylor PR. Monocyte and macrophage heterogeneity. Nat Rev Immunol. 2005;5(12):953-964.

37. Nguyen MT, et al. A subpopulation of macrophages infiltrates hypertrophic adipose tissue and is activated by free fatty acids via Toll-like receptors 2 and 4 and JNK-dependent pathways. J Biol Chem. 2007; 282(48):35279-35292.

38. Lumeng CN, Bodzin JL, Saltiel AR. Obesity induces a phenotypic switch in adipose tissue macrophage polarization. J Clin Invest. 2007;117(1):175-184.

39. Kosteli A, et al. Weight loss and lipolysis promote a dynamic immune response in murine adipose tissue. J Clin Invest. 2010;120(10):3466-3479.

40. Shoelson SE. Banking on ATM as a new target in metabolic syndrome. Cell Metab. 2006;4(5):337-338.

41. Bronte V, Serafini P, Mazzoni A, Segal DM, Zanovello $P$. L-arginine metabolism in myeloid cells controls T-lymphocyte functions. Trends Immunol. 2003; 24(6):302-306

42. Satriano J. Arginine pathways and the inflammatory response: interregulation of nitric oxide and polyamines: review article. Amino Acids. 2004; 26(4):321-329.

43. Patsouris D, Li PP, Thapar D, Chapman J, Olefsky JM, Neels JG. Ablation of CD11c-positive cells normalizes insulin sensitivity in obese insulin resistant animals. Cell Metab. 2008;8(4):301-309.

44. Granneman JG, Li P, Zhu Z, Lu Y. Metabolic and cellular plasticity in white adipose tissue I: effects of beta3-adrenergic receptor activation. Am J Physiol Endocrinol Metab. 2005;289(4):E608-E616.

45. Attie AD, Scherer PE. Adipocyte metabolism and obesity. J Lipid Res. 2009;50 suppl:S395-S399.

46. Rutkowski JM, Davis KE, Scherer PE. Mechanisms of obesity and related pathologies: the macro- and microcirculation of adipose tissue. FEBS J. 2009 276(20):5738-5746

47. Halberg N, Wernstedt-Asterholm I, Scherer PE. The adipocyte as an endocrine cell. Endocrinol Metab Clin North Am. 2008;37(3):753-768.

48. Spalding KL, et al. Dynamics of fat cell turnover in humans. Nature. 2008;453(7196):783-787.

49. Rodeheffer MS, Birsoy K, Friedman JM. Identification of white adipocyte progenitor cells in vivo. Cell. 2008;135(2):240-249.

50. Marques BG, Hausman DB, Martin RJ. Association of fat cell size and paracrine growth factors in development of hyperplastic obesity. Am J Physiol. 1998;275(6 pt 2):R1898-R1908

51. Cartwright MJ, Tchkonia T, Kirkland JL. Aging in adipocytes: potential impact of inherent, depot-specific mechanisms. Exp Gerontol. 2007;42(6):463-471.

52. Wang ZW, Pan WT, Lee Y, Kakuma T, Zhou YT, Unger $\mathrm{RH}$. The role of leptin resistance in the lipid abnormalities of aging. FASEB J. 2001;15(1):108-114

53. Karagiannides I, et al. Increased CUG triplet repeat-binding protein-1 predisposes to impaired adipogenesis with aging. J Biol Chem. 2006; 281(32):23025-23033.

54. Unger RH. Longevity, lipotoxicity and leptin: the adipocyte defense against feasting and famine. Biochimie. 2005;87(1):57-64.

55. Slawik M, Vidal-Puig AJ. Lipotoxicity, overnutrition and energy metabolism in aging. Ageing Res Rev. 2006;5(2):144-164

56. Nishimura S, et al. CD8+ effector T cells contribute to macrophage recruitment and adipose tissue inflammation in obesity. Nat Med. 2009;15(8):914-920.

57. Winer $\mathrm{S}$, et al. Normalization of obesity-associated insulin resistance through immunotherapy. Nat Med. 2009;15(8):921-929.

58. Liu J, et al. Genetic deficiency and pharmacological stabilization of mast cells reduce diet-induced obesity and diabetes in mice. Nat Med. 2009;15(8):940-945.

59. Boehm T, Folkman J, Browder T, O'Reilly MS. Antiangiogenic therapy of experimental cancer does not induce acquired drug resistance. Nature. 1997; 390(6658):404-407.

60 . Folkman J. Angiogenesis and angiogenesis inhibition: an overview. EXS. 1997;79:1-8.

61. Folkman J. Addressing tumor blood vessels. Nat Biotechnol. 1997;15(6):510.

62. Wong AL, Haroon ZA, Werner S, Dewhirst MW, Greenberg CS, Peters KG. Tie2 expression and phosphorylation in angiogenic and quiescent adult tissues. Circ Res. 1997;81(4):567-574.

63. Silverman KJ, et al. Angiogenic activity of adipose tissue. Biochem Biophys Res Commun. 1988; 153(1):347-352.

64. Cao Y. Angiogenesis modulates adipogenesis and obesity. J Clin Invest. 2007;117(9):2362-2368.

65. Lijnen HR. Angiogenesis and obesity. Cardiovasc Res. 2008;78(2):286-293.

66. Cao Y. Adipose tissue angiogenesis as a therapeutic target for obesity and metabolic diseases. Nat Rev Drug Discov. 2010;9(2):107-115.

67. Rupnick MA, et al. Adipose tissue mass can be regulated through the vasculature. Proc Natl Acad Sci US A. 2002;99(16):10730-10735.

68. Villaret A, et al. Adipose tissue endothelial cells from obese human subjects: differences among depots in angiogenic, metabolic, and inflammatory gene expression and cellular senescence. Diabetes. 2010; 59(11):2755-2763.

69. Vona-Davis L, Rose DP. Angiogenesis, adipokines and breast cancer. Cytokine Growth Factor Rev. 2009; 20(3):193-201.

70. Bell LN, et al. Adipose tissue production of hepatocyte growth factor contributes to elevated serum HGF in obesity. Am J Physiol Endocrinol Metab. 2006;291(4):E843-E848

71. Rehman J, et al. Secretion of angiogenic and antiapoptotic factors by human adipose stromal cells. Circulation. 2004;109(10):1292-1298.

72. Vona-Davis L, Rose DP. Adipokines as endocrine, paracrine, and autocrine factors in breast cancer risk and progression. Endocr Relat Cancer. 2007; $14(2): 189-206$

73. Brakenhielm E, et al. Adiponectin-induced antiangiogenesis and antitumor activity involve caspasemediated endothelial cell apoptosis. Proc Natl Acad Sci US A. 2004;101(8):2476-2481.

74. Ribatti D. The history of angiogenesis inhibitors. Leukemia. 2007;21(8):1606-1609.

75. Landskroner-Eiger S, et al. Proangiogenic contribution of adiponectin toward mammary tumor growth in vivo. Clin Cancer Res. 2009;15(10):3265-3276.

76. Papetti M, Herman IM. Mechanisms of normal and tumor-derived angiogenesis. Am J Physiol Cell Physiol. 2002;282(5):C947-970.

77. Ribatti D, Vacca A, Nico B, Presta M, Roncali L. Angiogenesis: basic and clinical aspects. Ital J Anat Embryol. 2003;108(1):1-24.

78. Zhang QX, Magovern CJ, Mack CA, Budenbender KT, Ko W, Rosengart TK. Vascular endothelial 
growth factor is the major angiogenic factor in omentum: mechanism of the omentum-mediated angiogenesis. J Surg Res. 1997;67(2):147-154.

79. Liekens S, De Clercq E, Neyts J. Angiogenesis: regulators and clinical applications. Biochem Pharmacol. 2001;61(3):253-270.

80. Claffey KP, Wilkison WO, Spiegelman BM. Vascular endothelial growth factor. Regulation by cell differentiation and activated second messenger pathways. J Biol Chem. 1992;267(23):16317-16322.

81. Tam J, Duda DG, Perentes JY, Quadri RS, Fukumura D, Jain RK. Blockade of VEGFR2 and not VEGFR1 can limit diet-induced fat tissue expansion: role of local versus bone marrow-derived endothelial cells. PLoS One. 2009;4(3):e4974

82. Fukumura D, et al. Paracrine regulation of angiogenesis and adipocyte differentiation during in vivo adipogenesis. Circ Res. 2003;93(9):e88-e97.

83. Davis $S$, et al. Isolation of angiopoietin-1, a ligand for the TIE2 receptor, by secretion-trap expression cloning. Cell. 1996;87(7):1161-1169.

84. Maisonpierre PC, et al. Angiopoietin-2, a natural antagonist for Tie2 that disrupts in vivo angiogenesis. Science. 1997;277(5322):55-60.

85. Dumont DJ, Anderson L, Breitman ML, Duncan AM. Assignment of the endothelial-specific protein receptor tyrosine kinase gene (TEK) to human chromosome 9p21. Genomics. 1994;23(2):512-513.

86. Dumont DJ, et al. Dominant-negative and targeted null mutations in the endothelial receptor tyrosine kinase, tek, reveal a critical role in vasculogenesis of the embryo. Genes Dev. 1994;8(16):1897-1909.

87. Sato K, et al. Stimulation by thyroid-stimulating hormone and Grave's immunoglobulin G of vascular endothelial growth factor mRNA expression in human thyroid follicles in vitro and flt mRNA expression in the rat thyroid in vivo. JClin Invest. 1995; 96(3):1295-1302.

88. Suri C, et al. Increased vascularization in mice overexpressing angiopoietin-1. Science. 1998; 282(5388):468-471.

89. Lobov IB, Brooks PC, Lang RA. Angiopoietin-2 displays VEGF-dependent modulation of capillary structure and endothelial cell survival in vivo. Proc Natl Acad Sci U S A. 2002;99(17):11205-11210.

90. Xue Y, et al. FOXC2 controls Ang-2 expression and modulates angiogenesis, vascular patterning, remodeling, and functions in adipose tissue. Proc Natl Acad Sci U S A. 2008;105(29):10167-10172.

91. Kim JY, et al. Obesity-associated improvements in metabolic profile through expansion of adipose tissue. J Clin Invest. 2007;117(9):2621-2637.

92. Semenza GL. Targeting HIF-1 for cancer therapy. Nat Rev Cancer. 2003;3(10):721-732.

93. Pouyssegur J, Dayan F, Mazure NM. Hypoxia signalling in cancer and approaches to enforce tumour regression. Nature. 2006;441(7092):437-443.

94. Pouyssegur J, Mechta-Grigoriou F. Redox regulation of the hypoxia-inducible factor. Biol Chem. 2006;387(10-11):1337-1346.

95. Karpe F, Fielding BA, Ardilouze JL, Ilic V, Macdonald IA, Frayn KN. Effects of insulin on adipose tissue blood flow in man. J Physiol. 2002; 540(pt 3):1087-1093.

96. Karpe F, Fielding BA, Ilic V, Macdonald IA, Summers LK, Frayn KN. Impaired postprandial adipose tissue blood flow response is related to aspects of insulin sensitivity. Diabetes. 2002;51(8):2467-2473.

97. Brahimi-Horn MC, Chiche J, Pouyssegur J. Hypoxia signalling controls metabolic demand. Curr Opin
Cell Biol. 2007;19(2):223-229.

98. Rausch ME, Weisberg S, Vardhana P, Tortoriello DV. Obesity in C57BL/6J mice is characterized by adipose tissue hypoxia and cytotoxic $T$-cell infiltration. Int J Obes (Lond). 2008;32(3):451-463.

99. Yin J, Gao Z, He Q, Zhou D, Guo Z, Ye J. Role of hypoxia in obesity-induced disorders of glucose and lipid metabolism in adipose tissue. Am J Physiol Endocrinol Metab. 2009;296(2):E333-E342.

100. Napolitano L. The differentiation of white adipose cells. An electron microscope study. J Cell Biol. 1963;18:663-679.

101. Chun TH, Hotary KB, Sabeh F, Saltiel AR, Allen $\mathrm{ED}$, Weiss SJ. A pericellular collagenase directs the 3-dimensional development of white adipose tissue. Cell. 2006;125(3):577-591.

102. Khan T, et al. Metabolic dysregulation and adipose tissue fibrosis: role of collagen VI. Mol Cell Biol. 2009; 29(6):1575-1591.

103. O’Hara A, Lim FL, Mazzatti DJ, Trayhurn P. Microarray analysis identifies matrix metalloproteinases (MMPs) as key genes whose expression is up-regulated in human adipocytes by macrophage-conditioned medium. Pflugers Arch. 2009;458(6):1103-1114.

104.Berria R, et al. Increased collagen content in insulin-resistant skeletal muscle. Am J Physiol Endocrinol Metab. 2006;290(3):E560-E565.

105. Muona P, et al. Hyperglycemic glucose concentrations up-regulate the expression of type VI collagen in vitro. Relevance to alterations of peripheral nerves in diabetes mellitus. Am J Pathol. 1993;142(5):1586-1597.

106. Maquoi E, Munaut C, Colige A, Collen D, Lijnen HR. Modulation of adipose tissue expression of murine matrix metalloproteinases and their tissue inhibitors with obesity. Diabetes. 2002;51(4):1093-1101. 